\title{
Amorphous fraction quantification analysis performing the Rietveld method in mixtures of active pharmaceutical ingredient ciprofloxacin and microcrystalline cellulose.
}

\author{
B.A. Ramírez ${ }^{1}$, L. Bucio ${ }^{1}$ \\ ${ }^{1}$ Instituto de Física, Universidad Nacional Autónoma de México, Circuito de la Investigación \\ Científica s/n Ciudad Uiversitaria, Coyoacán, México, D.F., 04150, México, D.F. \\ email: bara219@gmail.com
}

\section{Abstract}

Pharmaceutical solids are able to exist in several forms: polymorphic crystalline and amorphous arrangements. They present differences in their physico- chemical properties, for instance, melting point, density, morphology, solubility and color. These characteristics may have an impact on the stability (physical and chemical), bioavailability and bioequivalence $^{1}$; e.g. amorphous substances are unstable than crystalline substances. Differences in degrees of drug crystallization affect chemical and physical stability, rather than crystalline polymorphism of the substance. Tablets are solids preparations of active pharmaceutical ingredients and additives, many of the additives commonly used are poorly crystalline or amorphous, determining the final solubility in the drug.

Ciprofloxacin is a broad-spectrum antiinfective agent of the fluoroquinolone class. Ciprofloxacin has in vitro activity against a wide range of gram-negative and gram-positive microorganisms. Microcrystalline cellulose had been reported as an adhitive for ciprofloxacine tablets, following the pharmaceutical procedures of mixing and pressing. The next work develops an amorphous fraction quantification analysis performing the Rietveld method in mixtures of the active pharmaceutical ingredient ciprofloxacin (API-Cipro) and microcrystalline cellulose (MC) in different concentrations $(100 \% \mathrm{CIP}, 100 \% \mathrm{MC}, 90 \% \mathrm{CIP}$ 10\%MC, 75\%CIP-25\%MC, and 50\%CIP-50\% MC). Ciprofloxacin and microcrystalline cellulose were refined applying the Rietveld method taking into account the microstructure parameters only. The instrumental parameters were obtained using LaB6 as reference standard. The background of the diffraction patterns were modeled as a contribution of: (i) Thermal Diffuse Scattering (TDS) of the present crystalline phases; (ii) Air scattering.

\section{References}

[1] Norman Chieng, Thomas Rades, Jaakko Aaltonen. An overview of recent studies on the analysis of pharmaceutical polymorphs, Journal of Pharmaceutical and Biomedical Analysis 55 (2011) 618-644 